\title{
LA UNIVERSIDAD DE GRANADA Y LA ESCULTURA PÚBLICA
}

\author{
YOLANDA GUASCH MARÍ | UNIVERSIDAD DE GRANADA
}

ORCID iD: 0000-0001-9447-065X

\section{RAFAEL LÓPEZ GUZMÁN | UNIVERSIDAD DE GRANADA}

ORCID iD: 0000-0002-6966-6682

\begin{abstract}
RESUMEN
Este texto tiene como objetivo hacer un recorrido por la escultura pública relacionada con la Universidad de Granada, bien sita dentro de sus campus, en espacios urbanos limítrofes o relacionados con la actividad universitaria de la ciudad. La percepción de esta selección de obras permite al espectador realizar itinerarios con valores pedagógicos relacionados con la memoria histórica, así como con la estética plasmada por los artistas que las realizaron. Su diseño variado responde a las diversas tendencias artísticas desarrolladas desde la segunda mitad del siglo XX dominadas por la figuración, aunque no faltan obras de enorme expresividad y abstractas en su concepción. Para su visualización encontramos enlaces en las notas correspondientes.
\end{abstract}

PALABRAS CLAVE

Escultura pública, Universidad de Granada, Carlos V, Itinerarios, Memoria Histórica.

\section{THE UNIVERSITY OF GRANADA AND PUBLIC SCULPTURE}

\begin{abstract}
The aim of this text is to take a tour of the public sculpture related to the University of Granada, both located within its campuses and in neighboring urban spaces, as well as those related to the university activity in the city. The perception of this selection allows the viewer to make itineraries with pedagogical values related to historical memory, including the aesthetics captured by the artists who made them. The varied design corresponds to the different artistic tendencies since the second half of the 20th century, dominated by figuration, although there is no lack of works of enormous expressiveness and abstract in its conception. For its visualization, links can be found in the corresponding notes.
\end{abstract}

\section{KEYWORDS}

Public sculpture, University of Granada, Carlos V, Itineraries, Historic memory. 

a Universidad como "espacio del saber" se ha concretado a lo largo de la historia, desde su concepción occidental en el medioevo, a través de una arquitectura inserta en las ciudades que respondía a unas conceptualizaciones de diseño especiales atentas a las funciones a las cuales debía responder ${ }^{1}$. No es casual, por tanto, que aquellas urbes donde la universidad se ha convertido en una de sus señas de identidad, el desarrollo urbanístico y social haya estado vinculado al propio del establecimiento educativo. Esto sucede de forma parcial, al centrarse históricamente en barrios concretos, en ciudades como Bolonia (barrio de San Donato) o París (barrio latino), pero fagocitan la significación de las poblaciones pequeñas con el tiempo como sucedió en el caso de Oxford y Cambridge, hoy identificadas casi con exclusividad por sus respectivas universidades. En el ámbito norteamericano, incluso, las primeras universidades surgieron como campus aislados en la naturaleza generando, a posteriori, su propia ciudad ${ }^{2}$. Notorios son los casos españoles de Salamanca ${ }^{3}$, donde se imbrica, desde su origen en 1218, la ciudad y la universidad, o Alcalá de Henares ${ }^{4}$, fundación del Cardenal Cisneros en 1495 con un diseño de campus exento. Igualmente, en el caso de Granada el desarrollo de la universidad, que comienza su recorrido en el siglo XVI, ha condicionado la esencia de la ciudad así como su urbanismo, sobre todo en la ampliación de campus en el último tercio del siglo $\mathrm{XX}^{5}$.

Ahora bien, dentro de los espacios universitarios, en los límites entre las edificaciones de los campus o bien en las fronteras con el resto de la ciudad, se crean lugares de paso, de convivencia e interrelación que se urbanizan de diversa forma, siendo característico en algunos puntos la presencia de esculturas públicas con referentes simbólicos siempre añadidos. El interés de las universidades por estas obras y sus valores estéticos ha posibilitado su inclusión dentro de sus propios inventarios y catálogos, así como integrarlas en las políticas particulares de conservación y restauración.

1 Sobre la evolución de la arquitectura universitaria, Cfr. BONET CORREA, Antonio. La Arquitectura y el urbanismo de la Universidad. En: CIAN. Revista de historia de las universidades. 2014, nº 1, Vol. 17, pp. 23-30. ISSN 1139-6628.

2 El ejemplo más significativo es la fundación por el presidente Thomas Jefferson de la Universidad de Virginia en Charlottesville, construida entre 1814 y 1826 por el arquitecto Benjamín Henry Latrobe.

3 Cfr. ÁLVAREZ VILLAR, Julián. La Universidad de Salamanca. Arte Y Tradiciones. Salamanca: Universidad, 1973 (2a edición). ISBN 978-8460058-95-3.

4 Cfr. CASTILlO OREJA, Miguel Ángel. Ciudad, función $\gamma$ símbolos. Alcalá de Henares. Un modelo urbano de la España moderna. Alcalá de Henares. Col. Alcalá Ensayo, 1982. ISBN 978-8450079-94-2.

5 Cfr. HENARES CUÉLLAR, Ignacio y LÓPEZ GUZMÁN, Rafael. (eds.). Universidad $\gamma$ Ciudad. La Universidad en la historia $\gamma$ la cultura de Granada. Granada. Universidad de Granada, 1994. ISBN 978-84338-19-4. 
En este texto pretendemos presentar algunas de las esculturas situadas en los campus universitarios de la ciudad de Granada, trabajo que realizado originalmente en el marco de un proyecto I+D+i con el título "Patrimonio mueble urbano en Andalucía" (HAR-2012-38510), del que fue investigador principal Juan Antonio Arenillas Torrejón ${ }^{6}$, no tuvo el desarrollo previsto por las razones que expondremos ${ }^{7}$. Este proyecto venía a ampliar otras investigaciones similares anteriores, que habían servido de modelo metodológico, referentes a la ciudad de Sevilla bajo la dirección del citado Juan Arenillas y de Luis F. Martínez Montiel ${ }^{8}$. El objetivo general del estudio era implementar y definir una metodología de acercamiento a los espacios urbanos y su significación partiendo del análisis del mobiliario monumental, bien de carácter escultórico, de equipamientos u ornamentales. Bienes que enriquecen puntualmente la ciudad pero que se convierten en básicos para la percepción de esta y, sobre todo, de su entorno. En el citado proyecto se incluían investigadores de distintas provincias andaluzas, siendo Granada nuestra responsabilidad ${ }^{9}$. Para llevarlo a cabo se evaluaron razones de carácter conmemorativo que sirvieran de aglutinante para la selección del mayor número de esculturas ubicadas en la ciudad y que a la vez fueran representativas de su historia y valores sociales. Curiosamente en Granada, ciudad universitaria por antonomasia, no fue el nexo universitario uno de los elegidos, sino que se optaron por otros más perceptibles y singulares como fueron: El agua y su mobiliario urbano, La ciudad piensa sobre sí misma, Religiosidad y El Grafiti ${ }^{10}$. Aunque la universidad se trabajó como tema secundario, ya que había sido preseleccionado, quedando excluido finalmente ${ }^{11}$.

6 ARENILLAS TORREJÓN, Juan Antonio y MARTÍNEZ MONTIEL, Luis Francisco. Patrimonio mueble urbano de Andalucía. Patrimonio mueble urbano de Andalucía. En: Revista IAPH. [en línea]. 2013, nº 84, pp. 188-207. Disponible en: http://www.iaph.es/revistaph/index.php/revistaph/issue/view/85 [consulta: 6 de diciembre de 2020].

7 El proyecto se integraba dentro de las acciones de investigación propias del Instituto Andaluz de Patrimonio Histórico (IAPH).

8 ARENILLAS TORREJÓN, Juan Antonio y MARTÍNEZ MONTIEL, Luis Francisco. La imagen proyectada de Sevilla a través de los monumentos conmemorativos. En FERNÁNDEZ-BACA CASARES, R., FERNÁNDEZ CACHO, S. y SALMERÓN ESCOBAR, P. (dirs.). Guía del paisaje histórico urbano de Sevilla. Sevilla, Instituto Andaluz de Patrimonio Histórico. Sevilla: Consejería de Cultura, 2015, pp. 265-276. Este texto está realizado sobre trabajos anteriores de ambos investigadores como el denominado "La construcción del espacio urbano: monumentos públicos, mobiliario y equipamiento (Proyecto Guía del Paisaje Histórico urbano de Sevilla)" realizado en el año 2010 y que podemos consultar en los Activos Digitales IAPH: https://repositorio.iaph.es/handle/11532/326302 [consulta: 6 de diciembre de 2020].

9 El grupo de trabajo que se responsabilizó del análisis de la escultura pública en la ciudad de Granada estaba compuesto por María Encarnación Cambil Hernández, Guadalupe Romero Sánchez, Yolanda Guasch Marí y Rafael López Guzmán.

10 Los resultados de este se pueden consultar en Activos Digitales IAPH: https://repositorio.iaph.es/ handle/11532/332002 [consulta: 6 de diciembre de 2020].

11 Una vez realizado el trabajo de campo nos dimos cuenta de la imbricación tan intensa existente entre la Universidad y la ciudad de tal manera que los elementos conmemorativos universitarios proyectados en los ámbitos públicos eran asumidos como parte de la ciudad, decidiendo la eliminación de la línea e integrándola en otra más genérica, "la ciudad piensa sobre sí misma", ya que en Granada no se entiende la ciudad sin la universidad. Eso no quita que el punto de vista exclusivo desde la universidad no tenga su interés, de ahí la elaboración de este artículo. 
No obstante, consideramos que tiene suficiente entidad por la presencia social, histórica, urbanística y de percepción contemporánea para tenerlo en cuenta; razón por la que hemos decidido estudiar esta línea en este trabajo ${ }^{12}$.

\section{ITINERARIO APROXIMATIVO}

La universidad a nivel conmemorativo podríamos decir que funciona de forma independiente a los procesos de ocupación con este tipo de bienes en la ciudad, sobre todo atendiendo a que los comitentes no son los poderes públicos, sino que la universidad se glorifica a sí misma en sus espacios universitarios tanto en el interior de los edificios como en jardines y espacios de ocio que representan los distintos campus universitarios. De hecho son varios los elementos con carácter escultórico o de mobiliario público que indican la presencia de la universidad en la ciudad, marcando sus respectivos campus.

En un recorrido valorativo de las principales obras situadas en los recintos universitarios debemos comenzar con el busto de don Mariano del Amo y Mora (1809-1896) ubicado en el Jardín Botánico ${ }^{13}$ desde 1998, junto a la Facultad de Derecho, y con acceso directo desde el viario urbano. La escultura es una copia de la realizada por José Navas Parejo ${ }^{14}$ que retrata al primer catedrático de Botánica de la Universidad que, además, fue decano de la Facultad de Farmacia ${ }^{15}$. También dentro del Jardín encontramos un monolito de piedra sobre el que se sitúa un busto de bronce fundido que representa a don Fernando de los Ríos ${ }^{16}$, el cual ejerció como catedrático de Teoría Política en la Facultad de Derecho, aneja a este espacio.

12 Existe también una razón al margen de lo científico como es que algunas de estas conclusiones se expusieron en una conferencia que se organizó dentro de un curso de la Universidad de Cádiz con el título "Nuevos métodos de valoración del mobiliario urbano: el caso de Andalucía", donde se exponían resultados del proyecto de investigación citado. Uno de los autores de este texto, Rafael López Guzmán, impartióla conferencia titulada “Granada. Universidad y Ciencia" (3 de julio de 2015). El hecho de que la publicación sea en homenaje a dos insignes gaditanos y amigos, Juan Ramón Cirici Narváez y Fernando Pérez Mulet, nos llevó a pensar que era el tema adecuado para esta colaboración.

13 Cfr. sculturayarte.com/025315/Mariano-del-Amo-en-Granada.html\#.X98HY-CCE3k [consulta: 20 de diciembre de 2020].

14 El escultor José Navas-Parejo (1883-1953), aunque originario de Alora (Málaga), desarrolló la casi totalidad de su vida en Granada. Escultor prolífico al frente de un importantísimo taller centrado en obras de temáticas religiosas, funerarias, conmemorativas y retratos. También se desarrolló como orfebre.

15 La obra original se presentó, fuera de concurso, a la Exposición de Bellas Artes de Granada en 1910, obteniendo un Primer Diploma. La universidad conserva dos réplicas de esta. La primera es la ubicada en el Jardín Botánico, la segunda se encuentra en el vestíbulo de la Facultad de Farmacia. Cfr. https://atalaya3d.ugr.es/obra/mariano-delamo-y-mora [consulta: 20 de diciembre de 2020].

16 Cfr. https://tropter.com/es/espana/granada/la-universidad-de-granada-botanical-garden?gid=1\&pid=96237 [consulta: 20 de diciembre de 2020]. 
La obra fue realizada por el artista José Noja ${ }^{17}$ en 1980, siendo financiada por el grupo parlamentario socialista de Granada, en tanto que el homenajeado fue militante de este partido político ocupando varios cargos de gobierno antes del golpe militar de 1936.

Inmediato al Jardín Botánico, como ya lo hemos indicado, se encuentra la Facultad de Derecho, en cuyo patio principal, encontramos una escultura de medio cuerpo dedicada al Padre Francisco Suárez ${ }^{18}$, obra del escultor Francisco Martínez Olaya ${ }^{19}$, fechada en $1953^{20}$. Las razones de su presencia estriban en las aportaciones en el ámbito de la Filosofía del Derecho del reconocido teólogo, filósofo y jurista.

De carácter estético, pero valorativo, en este caso no del personaje representado sino de la propia artista que realiza la obra, tenemos la escultura que con el sencillo título de "La Danza" $^{21}$ de la granadina Carmen Jiménez ${ }^{22}$ y que encontramos en la entrada del Hospital Real de Granada, sede del rectorado de nuestra universidad. Se trata de una figura femenina en bronce, de tamaño natural, desnuda, con las manos cruzadas por la espalda, de cabellos rizados hacia atrás con el rostro mirando hacia la derecha; se dispone en forzado contraposto con la pierna derecha más adelantada y la izquierda doblada hacia atrás. La expresión de la figura resulta un tanto hierática y el modelado muy blando, pero de buena factura. Se trata de una copia en bronce de la obra original realizada, posiblemente, en terracota o en resina de poliéster, cuyo paradero se desconoce ${ }^{23}$.

17 José Noja Ortega (Aracena, 1938) es un prolífico artista (pintor y escultor) que combina su actividad creativa con otras acciones como piloto de aviación civil e, incluso, futbolista. De hecho, jugó en los años 60 del siglo pasado en el Ajax de Ámsterdam.

18 La importancia de este intelectual y teólogo granadino hace que su conmemoración en Granada salga de los espacios universitarios para ocupar, también, una hornacina en la plaza de las Pasiegas; en un chaflán realizado a propósito en el antiguo edificio de la Universidad. Este nicho fue erigido en 1917 por el escultor José Navas-Parejo en el ámbito de conmemoración del III Centenario de su muerte. El relieve se enmarca por una estructura arquitectónica, a modo de vano, que retoma los elementos compositivos renacentistas de la portada principal del edificio.

19 Cfr. https://atalaya3d.ugr.es/obra/monumento-al-padre-francisco-suarez [consulta: 20 de diciembre de 2020].

20 Cfr. https://atalaya3d.ugr.es/obra/monumento-al-padre-francisco-suarez [consulta: 28 de noviembre de 2020].

21 Cfr. https://es.m.wikipedia.org/wiki/Archivo:La_Danza._Carmen_Jimenez.1975.jpg [consulta: 20 de diciembre de 2020].

22 Carmen Jiménez (1920-2016) fue una reconocida escultora, natural de La Zubia (Granada), que trabajó fundamentalmente en Sevilla donde desarrolló una amplia actividad docente en la Facultad de Bellas Artes de esa ciudad.

23 Los datos corresponden al inventario de patrimonio de la Universidad de Granada. https://patrimonio.ugr.es/ obra-del-mes/la-danza/ [consulta: 28 de noviembre de 2020]. 
Completa esta entrada del Hospital Real una escultura de María Lagunes ${ }^{24}$, artista mexicana muy relacionada con la Universidad de Granada, realizada en 2004 en acero al carbón, situada aquí en relación con una gran exposición sobre su obra realizada en el interior del Hospital Real el mismo año. La obra se titula "Estudio para escultura urbana" ${ }^{25}$. Una obra donde priman los valores geométricos, marcando ejes verticales interrumpidos por salientes trapecios que permiten una lectura constante entre materia y espacio. La textura acerada mate permite la integración como obra pública en la atmósfera quieta y ajardinada del entorno.

En otro espacio universitario, también con valores paisajísticos, como es el Carmen de la Victoria, encontramos en una de sus fuentes un "Desnudo femenino" ${ }^{26}$ vaciado de poliéster, de $161 \mathrm{~cm}$. de altura, realizado por el escultor Francisco Rivas Carmona en 1989. El espacio donde se ubica se completa con una pequeña alberca y unas columnas de bronce fundido de arrastre, de $211 \mathrm{~cm}$. de altura, realizadas hacia 1900. También en los jardines del Carmen se encuentra una segunda e intimista fuente titulada "Sol y Luna" ${ }^{27}$ del artista Julio Juste ${ }^{28}$. Se trata de dos rostros situados en los extremos de un ángulo de $90^{\circ}$. Ambas caras, realizadas en bronce dorado, abren sus labios para conectarse mediante el pequeño chorro de agua. Las referencias al sol, como un ser ajado por la edad, y la luna, de aspecto joven, se unifican en la fuerza de la expresión vital de ambos. Esta obra, que hay que buscarla entre el frondoso jardín donde se ubica, aporta lirismo y preciosismo técnico que nos remite a las cualidades artísticas de su autor.

En otros espacios semipúblicos de la universidad, como es el amplio atrio que precede a la Facultad de Filosofía y Letras en el Campus de Cartuja, se ubica el busto de la poeta Elena Martín Vivaldi ${ }^{29}$. La obra se realizó dentro de un programa de actos conmemorativos del centenario del nacimiento dela poetisa.Seexponesobreunpedestal depiedra esta magnífica

24 Cfr. https://es.m.wikipedia.org/wiki/Archivo:Estudio_para_escultura_urbana._Mar\%C3\%ADa_Lagunes._2004.jpg [consulta: 20 de diciembre de 2020].

25 Cfr. https://patrimonio.ugr.es/obra-del-mes/estudio-para-escultura-urbana/ [consulta: 28 de noviembre de 2020].

26 Cfr. http://carmendelavictoria.ugr.es/pages/imagenes/_img/_mg_5267 [consulta: 20 de diciembre de 2020].

27 Cfr. https://atalaya3d.ugr.es/publicacion/obra-del-mes-en-la-ugr-sol-y-luna [consulta: 20 de diciembre de 2020].

28 Julio Juste (Beas de Segura, 1952-Granada, 2017), licenciado y doctor en Historia del Arte por la Universidad de Granada comienza su trayectoria artística en 1970. Diseñador, pintor, gestor cultural, polifacético artista que plasmó su creatividad en diversos soportes que irían desde el lienzo a proyectos de videoarte, siempre con una enorme reflexión teórica que, a veces, nos sorprende por la frescura de sus realizaciones y su capacidad de comprensión inmediata.

29 Cfr. https://www.pinterest.es/pin/355362226841430513/ [consulta: 20 de diciembre de 2020]. 
cabeza de bronce realizada en el año 2007 por el escultor Jesús Martínez Labrador, el cual tuvo la oportunidad de retomar y fundir un trabajo previo sobre la escritora que realizó cuando todavía vivía, posando directamente ${ }^{30}$. La relación de esta cabeza con su espacio es deficiente, pero también es lo que se persigue. El espacio estaba diseñado previamente y su ubicación en un lateral remite a la propia forma de ser de la homenajeada: recatada y no dada a las celebraciones ni primeros planos. No obstante, es un referente conocido por todos los universitarios que transitan por la Facultad de Filosofía y Letras.

\section{Imagen 1}

Busto del escritor Aleksandr S. Pushkin, 2015 (Foto RLG)

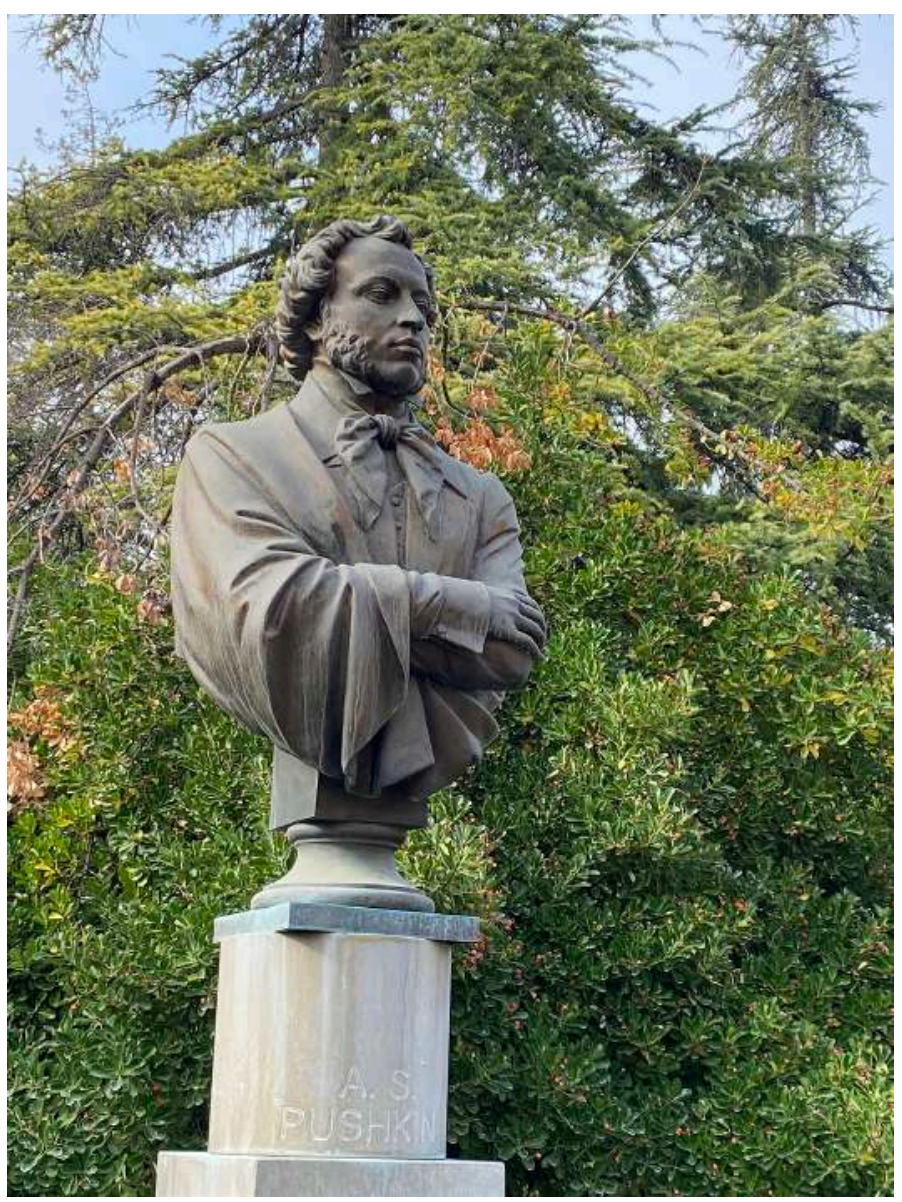

En el entorno del denominado campus de Fuentenueva encontramos esculturas, algunas muy recientes, de interés estético y simbólico evidentes. Nos estamos refiriendo, por ejem-

30 DURÁN, Blanca. El recuerdo en bronce de Martín Vivaldi preside ya "su Facultad". Granada Hop, 6 de diciembre, 2007. Cfr. https:// www.granadahoy.com/ocio/recuerdo-Martin-Vivaldi-preside-Facultad_0_101690095.html [consulta: 28 noviembre de 2020]. 
plo, al busto dedicado al escritor ruso Aleksandr S. Pushkin ${ }^{31}$ (IMAGEN No 1), considerado el iniciador de la moderna literatura rusa, el cual se ubicó en el año $2015^{32}$. La escultura en bronce, que se eleva sobre un complejo soporte de piedra de Elvira, fue realizada por el artista Boris Anatolievich Petrov ${ }^{33}$ siguiendo los modelos iconográficos del escritor derivados de pinturas del siglo XIX por lo que exhibe las características formales de estas con rasgos de claro romanticismo que acercan el personaje a su propia literatura ${ }^{34}$.

\section{Imagen 2 \\ Escultura de Emilio Herrera Linares, 2017 (Foto RLG)}

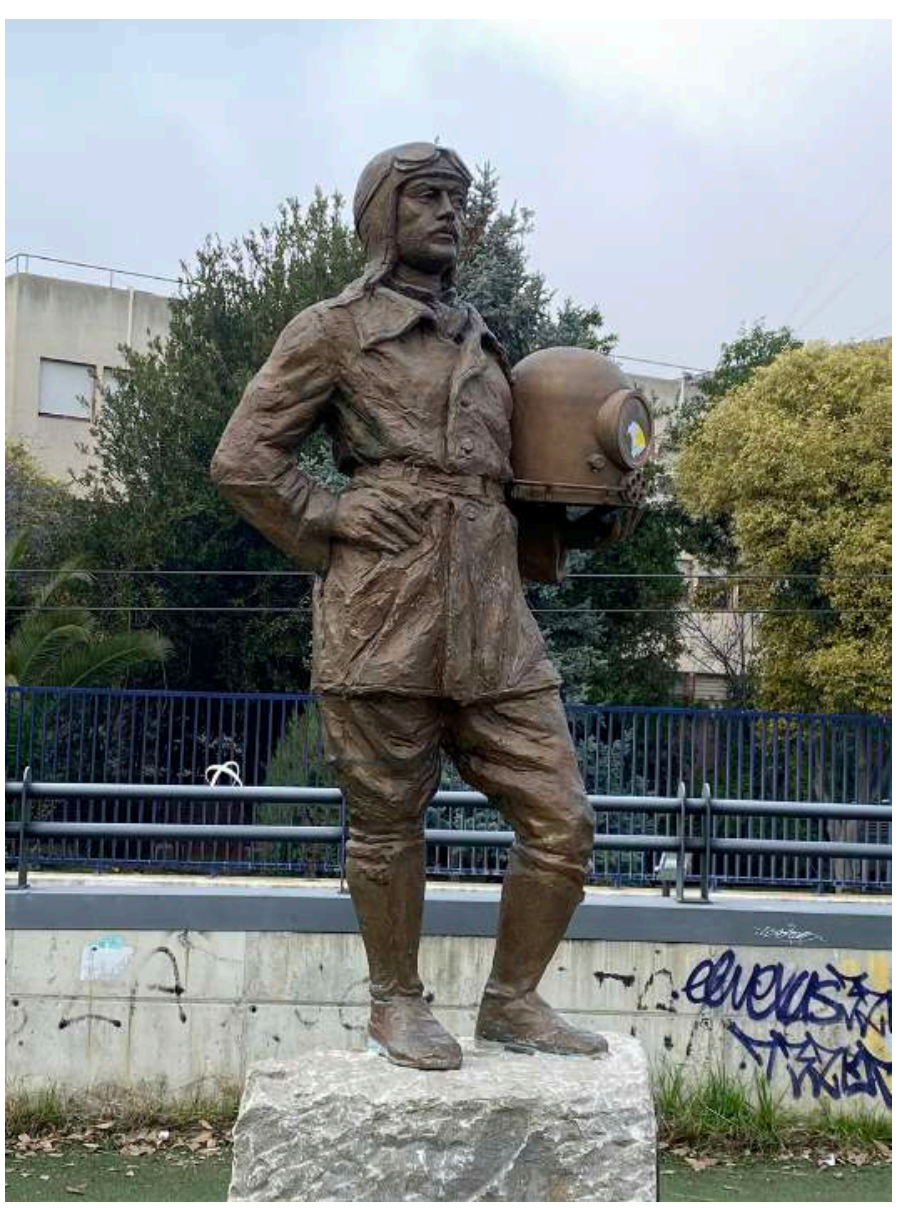

31 Aleksandr Serguéyevich Pushkin (1799-1837) cultivó tanto la poesía como la novela y el teatro. Enmarcado dentro de la corriente romántica, influiría en los escritores posteriores como Dostoievski, Gogol o Tolstoi.

32 En la inscripción situada al pie de la escultura se especifica que el monumento se ha erigido con motivo del XIII congreso de la Asociación Internacional de Profesores de Lengua y Literatura Rusas (MAPRYAL), celebrado en Granada entre los días 13 al 20 de septiembre de 2015, sumándose al homenaje al poeta la Universidad de Granada y, a nivel financiero, la Fundación "Russki Mir". Cfr. https://casarusia.com/wp-content/uploads/2015/11/20151119_143256.jpg [consulta: 20 de diciembre de 2020].

33 https://rinconesdegranada.com/escultura-aleksandr-pushkin [consulta: 5 de diciembre de 2020].

34 Ejemplo de esa relación iconográfica la podemos observar en el retrato del escritor realizado en 1827 por el pintor Orest Kiprenski conservado en la Galería Tretiakov de Moscú. 


\section{Imagen 3}

Portada del siglo XVIII junto a la Escuela Técnica Superior de Ingeniería de Edificación (Foto RLG)

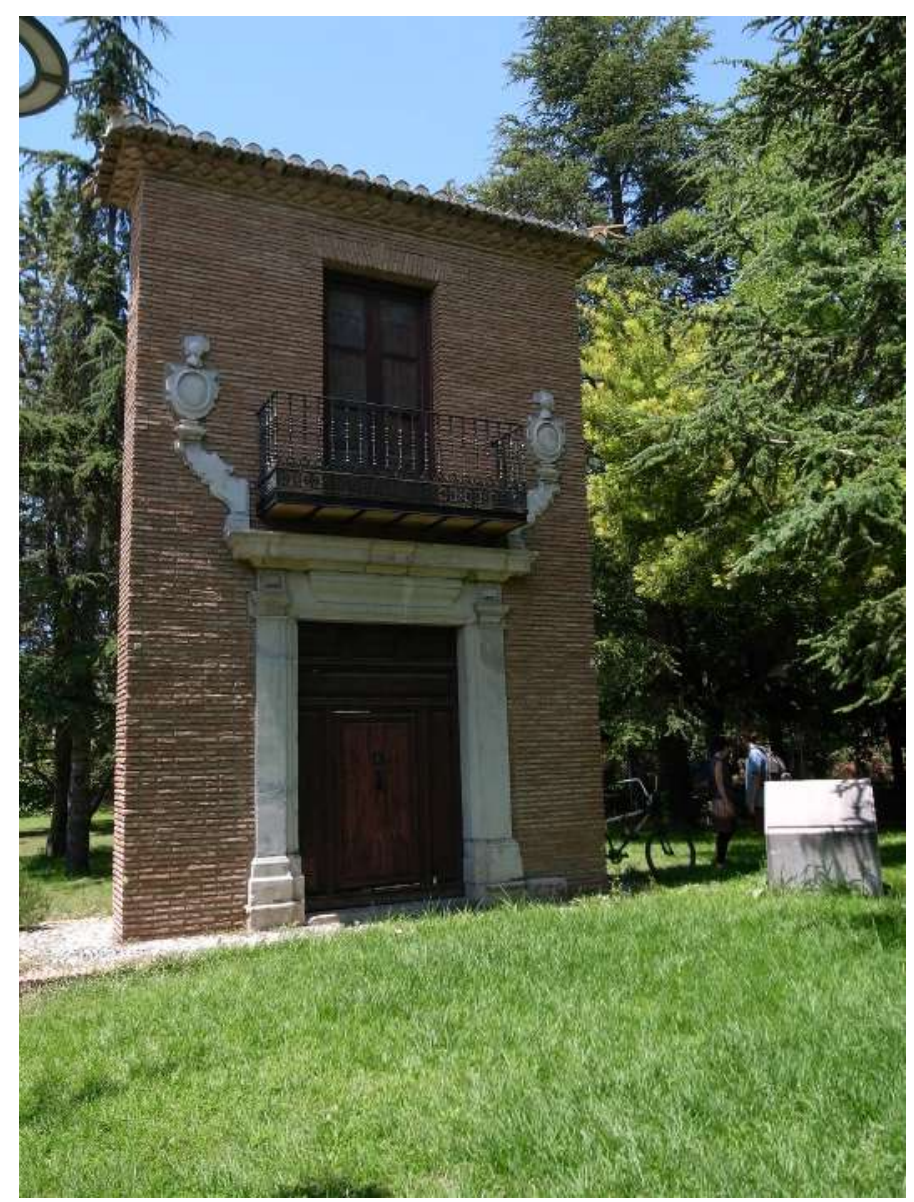

Sin salirnos del espacio de Fuentenueva nos encontramos con la escultura dedicada a Emilio Herrera Linares ${ }^{35}$ (IMAGEN No 2). Fue inaugurada en el año 2017, cincuentenario de su muerte, reconociendo las aportaciones científicas de este granadino olvidado por el régimen franquista. Es más, la financiación de la escultura se realizó por suscripción popular. El vaciado en bronce, obra de los artistas Balbino Montiano Benítez y Antonio Martínez Villa, representa, de cuerpo entero y a tamaño natural, al ingeniero militar con el traje de aviador. La mano derecha la apoya en la cadera a la vez que avanza la pierna izquierda restando estatismo a la obra. La izquierda sostiene una escafandra correspondiente al traje que inventó para ascensos a grandes alturas que fue base para el diseño de los trajes de

35 El granadino Emilio Herrera Linares (1879-1967) fue un reconocido ingeniero, científico y destacado aviador. Fiel a la República se exilió tras la Guerra Civil, participando en distintos gobiernos en el exilio,llegando a ser nombrado Presidente del Gobierno de la República Española (1960-1962). Entre sus propuestas de carácter científico estaría la escafandra autónoma pensada para los vuelos en globos a gran altura que sería el antecedente de los actuales trajes espaciales. 
astronautas. Un basamento pétreo sobre eleva la escultura otorgándole monumentalidad, incrementada por el gesto y la pose del aviador ${ }^{36}$.

En este itinerario estético, tenemos que incluir con otra valoración y percepción, la presencia en el entorno ajardinado de la Escuela Técnica Superior de Ingeniería de Edificación, de una perfecta anastilosis con una portada del siglo XVIII donada por las herederas de don Francisco Rivas Contreras y de don Miguel Giménez Yanguas a la Universidad en 1996 (IMAGEN No 3). Su ubicación en este lugar está perfectamente relacionada con la docencia que se imparte en las instalaciones contiguas.

En el ámbito específicamente científico cabe señalar algunos espacios verdes en torno a facultades que han servido y están sirviendo para concretar algunos elementos con valores escultóricos aunque con el objetivo de mantener la memoria de los avances técnicos. Es la razón, por ejemplo, de la presencia de una máquina de vapor procedente de la azucarera San Isidro fechada en 1901, de fabricación alemana que encontramos en la zona de entrada de la Facultad de Ciencias ${ }^{37}$.

Como resumen de este pequeño recorrido por los ámbitos universitarios de Granada, quiero cerrar con el monumento dedicado genéricamente a los investigadores, aunque concretado por su ubicación en la Facultad de Farmacia, el cual es obra del escultor José Fuentes . El espacio que ocupa es una zona marginal producida por la imbricación de los distintos volúmenes arquitectónicos que definen esta Facultad. Sobre un pequeño plinto se alza un pedestal en el que se ubica la figura de un hombre de escala superior a la humana. Vestido con una túnica que le llega hasta los pies, sujeta con su mano derecha un matraz. La cabeza en muy pequeña con relación al resto del cuerpo. En el lateral derecho lleva una placa donde se puede leer la inscripción: "A todos los que con su dedicación y estudio han contribuido a la ciencia" y, en el lateral opuesto, "Asociación de Antiguos Alumnos de la Facultad de Farmacia y la de Farmacéuticos de las Letras y las Artes rindieron homenaje al investigador con esta escultura"; y, en el frontal se lee "Al investigador"(IMAGEN No 4). Aunque bastante oculta, a nivel público por el lugar en que se ubica, es una obra de enorme contundencia por su monumentalidad y expresividad abstracta que no deja apenas resquicios para que la mirada se centre en el relato de la minuciosidad de la escultura al reducirse tanto los objetos anexos como la

36 https://rinconesdegranada.com/escultura-a-emilio-herrera-linares [consulta: 5 de diciembre de 2020].

37 Cfr. https://atalaya3d.ugr.es/obra/bomba-de-vapor-duplex-de-la-fabrica-azucarera-la-vega [consulta: 20 de diciembre de 2020]. 
propia estructura anatómica del personaje simbolizado. Opción contemporánea de una estética que huye del detalle anecdótico para componerse a partir del volumen y su expresividad manejada por el artista a través del punto de vista, desde abajo, por parte del espectador. Es una creación, por tanto, reseñable en la producción contemporánea de arte público.

\section{Imagen 4}

Escultura "Al Investigador", 1995 (Foto RLG)

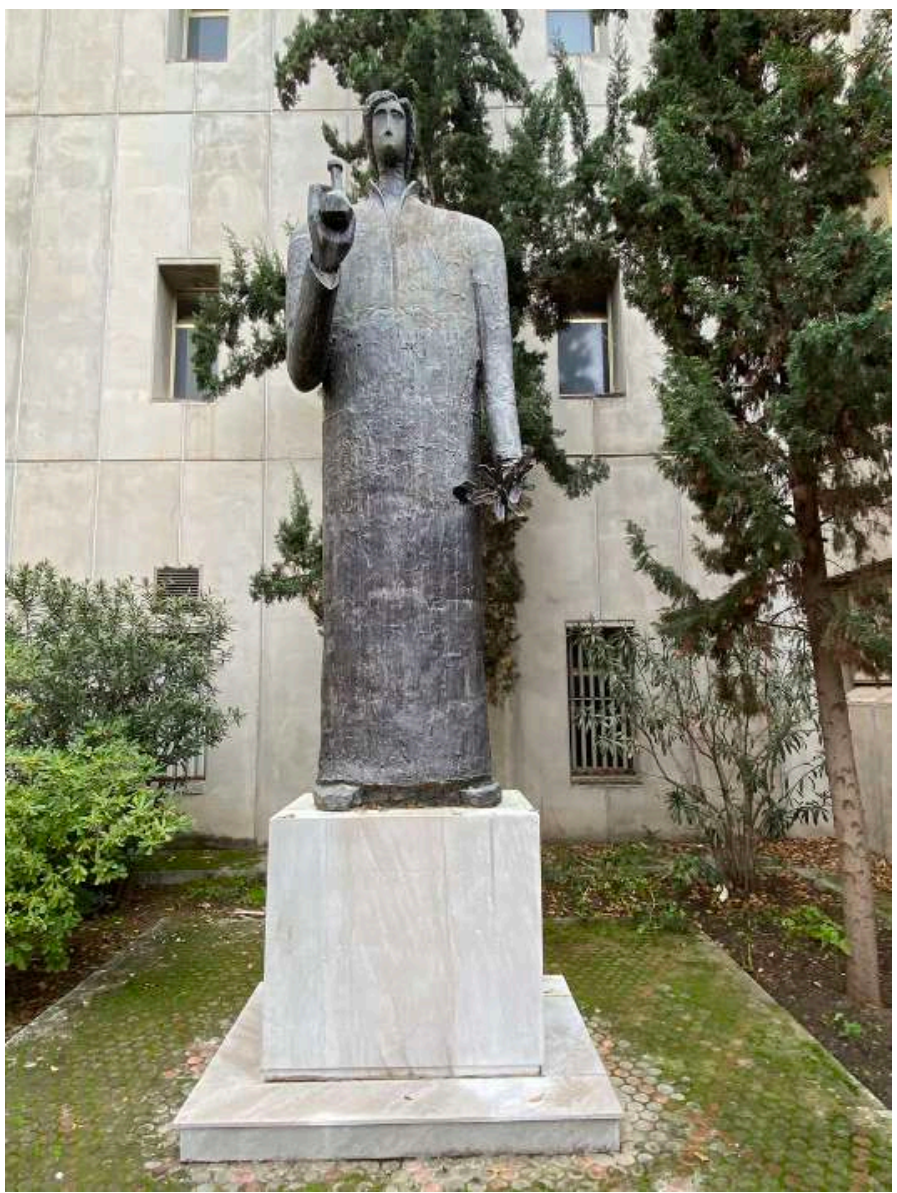

\section{SALIENDO DE LA UNIVERSIDAD}

Dada la importancia de la Universidad en la ciudad, se hace permeable la memoria derivada de la misma en la propia historia urbana. Valoración siempre con matizaciones ya que algunos universitarios trascienden a esa cualidad para integrarse en un concepto más amplio y universal, siendo el caso más paradigmático, en este sentido, la figura de Federico García Lorca. No obstante, hay personajes públicos asumidos por la ciudadanía que mantienen su horizonte comprensivo, fundamentalmente, como universitarios. 
Es el caso de Joaquina Eguaras (1897-1982), cuyo busto se sitúa en la denominada zona norte de la ciudad, dando nombre tanto a la calle como al barrio ${ }^{38}$. Esta insigne universitaria estudió Magisterio y Filosofía y Letras. En 1925 se convirtió en la primera mujer profesora universitaria, siendo la única hasta 1935. Compaginó su actividad como docente con su implicación en la Escuela de Estudios Árabes e, igualmente, fue directora del Museo Arqueológico. El busto de la insigne universitaria se sitúa sobre un pedestal vertical que apoya sobre una pequeña base cuadrangular. Los rasgos físicos nos acercan a sus últimos años de vida, con gesto serio y mirada firme. Una cartela presenta la siguiente inscripción: “GRANADA A JOAQUINA EGUARAS". El monumento está rodeado por cuatro fustes de columna. Se realizó en el año 2003 por los escultores M. Huertas y A.M. Villa ${ }^{39}$.

El trascender de los personajes universitarios por sus actividades, como ya hemos comentado, fuera de la órbita del tema que nos atañe, es la razón que permite destacar el conjunto escultórico dedicado a Fernando de los Ríos ${ }^{40}$, realizado en el año 2005 por los artistas José Manuel Darro y Alejandro Muñoz; duplicando el reconocimiento al insigne catedrático y político que ya había sido referenciado en el Jardín Botánico de la Universidad.

El espacio que ocupa, en este caso, es una rotonda en el nuevo diseño de la carretera de Armilla convertida en avenida urbana. Aquí, sobre una gran plataforma de bronce se representa a Fernando de los Ríos, con una escultura de cuerpo entero de 3,5 metros de altura, en actitud de pasear con un libro en una mano y una estilográfica en la otra, poniendo de manifiesto su perfil intelectual y humanista. El espacio se completa con un granado geométrico de $4 \mathrm{x} 4$ metros que recuerda las raíces locales del representado ${ }^{41}$.

También, duplicada en espacio universitario y urbano, valoramos, dentro de este apartado, la escultura dedicada a la poetisa Elena Martín vivaldi ${ }^{42}$ situada en el boulevard de la Avenida de la Constitución ${ }^{43}$. Un espacio escultórico donde conviven insignes personajes

38 Cfr. https://repositorio.iaph.es/bitstream/11532/318014/2/70_0132822.jpg [consulta: 20 de diciembre de 2020].

39 ROMERO SÁNCHEZ, Guadalupe y MARFIL-CARMONA, Rafael. La imagen de la mujer en el patrimonio urbano de Granada. El espacio público de la ciudad como "escenario comunicativo". En: Revista Internacional de Investigación en Comunicación aDResearch. ESIC, 2020, n²2, Vol. 22, p. 205.

40 Cfr. https://rinconesdegranada.com/escultura-a-fernando-de-los-rios [consulta: 20 de diciembre de 2020].

41 La escultura fue financiada por Caja Granada que tenía su sedecentral en la rotonda donde se ubica la escultura referida. 42 La poetisa Elena Martín Vivaldi (1907-1998) estudió Magisterio y, más tarde, Filosofía y Letras en la Universidad de Granada a la que siempre estuvo muy ligada desde su trabajo como bibliotecaria de las facultades de Medicina y, posteriormente, Farmacia. A lo largo de su vida recibió el reconocimiento a su obra a través de diversos premios. Fue nombrada Hija Predilecta de Granada (1988) y recibió la Medalla de la Real Academia de Bellas Artes, así como reconocimientos por parte de las facultades de Filosofía y Letras y de Farmacia.

43 Cfr. https://es.wikiloc.com/rutas-a-pie/2019-02-01-granada-fuente-gigantones-capilla-real-ptas-monaitaelviraparque-agua-cartuja-prision-pr-32870011/photo-21170636 [consulta: 20 de diciembre de 2020]. 
granadinos, desde el Gran Capitán al torero Frascuelo en un desorbitado programa escultórico ${ }^{44}$, precipitado en el tiempo y con distinta cualidad por parte de los artistas a los que se encargó. La obra que nos ocupa representa a la poetisa sentada en un banco con un libro abierto y utilizando como marcapáginas un ramillete de flores ${ }^{45}$. La escultura en bronce fundido es del año 2010 y su autor José Antonio Castro Vílchez ${ }^{46}$. El resultado invita al acompañamiento interior hacia los versos de una poeta y una universitaria ${ }^{47}$.

\section{Imagen 5}

\section{Carlos V en la batalla de Mülberg, 2000. Interpretación del equipo ROTO \\ (Foto RLG)}

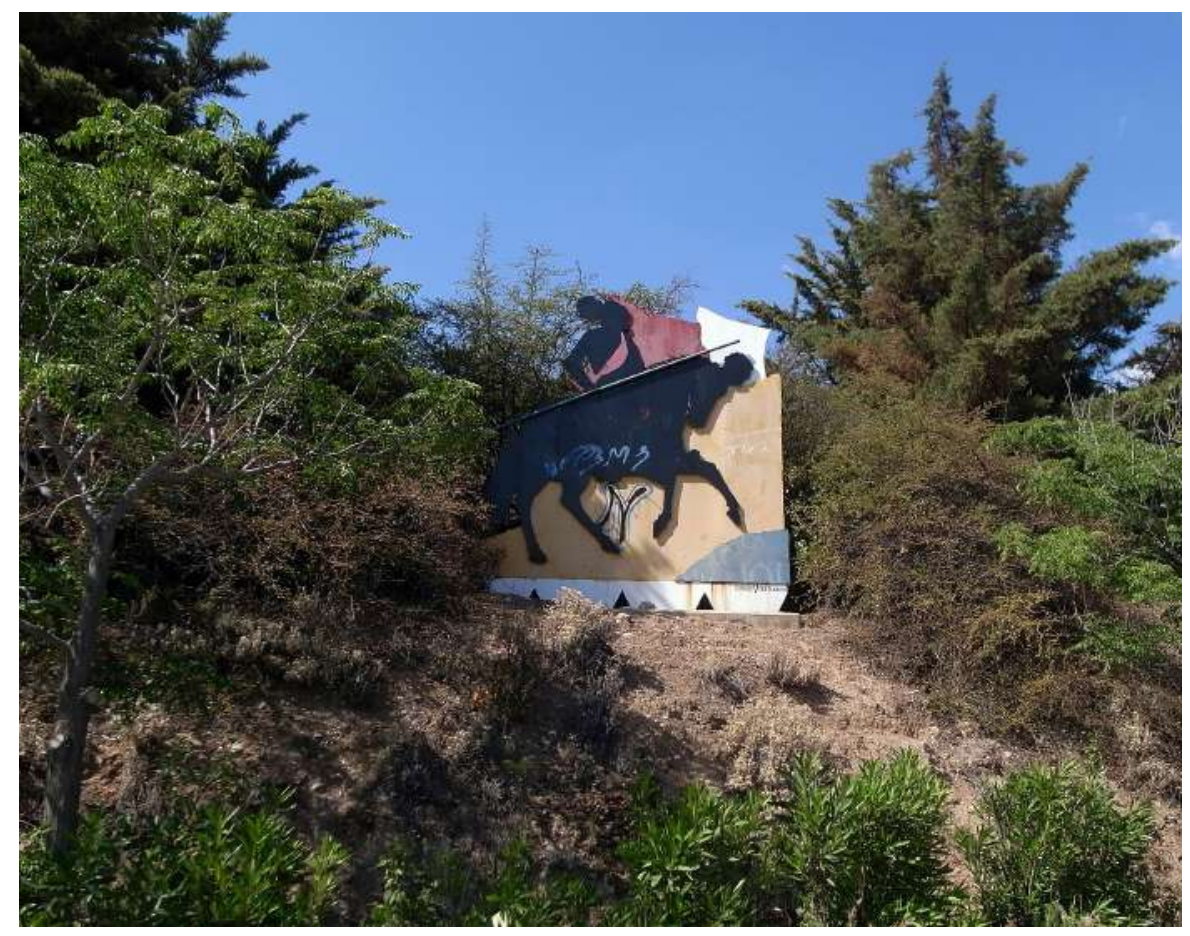

44 Se trató de hacer un proyecto conmemorativo partiendo de la elaboración por el periódico Ideal, en el año 2000 , de una lista de los 100 granadinos más relevantes del siglo XX. De estos se escogieron, inicialmente, a 10. Para la realización de las esculturas se eligieron a otros tantos artistas dándole total libertad en su ejecución, salvo algunas condiciones como las dimensiones que debían ser de cuerpo entero y tamaño natural, a excepción del busto del Gran Capitán que inicia el recorrido desde la Gran Vía.

45 La inspiración del proyecto parece que estuvo en la escultura sedente de Albert Einstein, obra de Miguel Barranco López de 1998, situada a las puertas del Parque de las Ciencias, la cual es una imagen fundida, de cuerpo entero, que aparece sentada en uno de los ángulos de un banco de metal, con el que forma conjunto, y que es un símbolo de esa institución, además de fotografía obligada de sus visitantes.

46 Este escultor nacido en Granada (1938) se formó en Sevilla, aunque volvió a su tierra natal donde intercala su docencia en la Facultad de Bellas Artes con la creación artística. Es académico numerario de la Real Academia de Bellas Artes de Nuestra Señora de las Angustias de Granada y correspondiente de la Real Academia de Bellas Artes de Santa Isabel de Hungría de Sevilla.

47 ROMERO SÁNCHEZ, Guadalupe. y MARFIL-CARMONA, Rafael. La imagen de la mujer... op.cit. p. 204. 
Es interesante, también, la proyección de la universidad en la ciudad, a través de sus eventos y su visualización pública. Me refiero al pebetero que se diseñó para los Juegos Universitarios (Universiada) ${ }^{48}$ de invierno celebrada en al año $2015^{49}$, organizados por la Federación Internacional de Deportes Universitarios (FISU). Pese a que la organización tuvo un rango fundamentalmente universitario, las instituciones municipales, provinciales y autonómicas, jugaron un importantísimo papel al igual que los responsables de Sierra Nevada. Tras el evento deportivo, el elemento simbólico del pebetero ${ }^{50}$ se utilizó como ornato de una de las rotondas de la carretera de Armilla, cercana a donde encontramos el grupo escultórico dedicado a Fernando de los Ríos.

\section{CARLOS V, FUNDADOR DE LA UNIVERSIDAD DE GRANADA}

La figura de Carlos V está, evidentemente, ligada a la memoria de la ciudad de Granada. Su estancia en la ciudad en 1526 fue larga y, además, muy importante para el devenir de esta. Actuaciones como la decisión de la construcción del Palacio en la Alhambra, las intervenciones y rehabilitaciones en el complejo palatino nazarí, la redefinición de la catedral, apenas comenzada, en panteón imperial, la puesta en funcionamiento del Hospital Real o la fundación de la Universidad, marcaron nuestro devenir histórico que mantiene su memoria a través de sus realizaciones y, sobre todo, mediante el funcionamiento de la universidad, tema que tratamos en este texto.

En relación con su presencia a través de la plástica pública tenemos que reseñar que en el año 2000 con motivo de la celebración en la ciudad de la exposición "Carlos V. Las Armas y las Letras" dentro de las actividades conmemorativas del Centenario de Felipe II y Carlos V (1999-2000) se realizó un recortable de Carlos V que reinterpreta de forma abstracta y colorista el cuadro de Tiziano que representa a Carlos V en la batalla de Mülberg por los diseñadores de Equipo RO-TO (Gerardo Rosales y Luis Torralba) (IMAGEN No 5).

Esta pieza, realizada en plancha de hierro policromada, que estuvo en el jardín del entorno del Hospital Real, se ha instalado definitivamente abriendo los accesos al campus de Cartuja. Interpretación vanguardista de la figura del emperador para espacios universitarios también contemporáneos.

\footnotetext{
48 https://rinconesdegranada.com/rotonda-universiada-2015 [consulta: 20 de diciembre de 2020].

49 Se trataba de la edición número XXVII.

50 Durante el evento deportivo el pebetero estuvo situado en el Paseo del Violón frente al Palacio de Exposiciones y Congresos. Cfr. https://rinconesdegranada.com/rotonda-universiada-2015 [consulta: 29 de noviembre de 2020].
} 
Pero la escultura más emblemática del emperador como fundador de la Universidad la tenemos situada en un espacio urbano, la plaza de la Universidad ante la Facultad de Derecho ${ }^{51}$. Se trata de una copia realizada en 1948 de la original, conservada en el Museo del Prado ${ }^{52}$, realizada en mármol de Carrara, fechada en torno a 1553, por los escultores Leone y Pompeo Leoni. Estatua de tamaño mayor que el natural representando al Emperador en pie, con media armadura y bastón de mando. El Toisón de Oro que pende del pecho, la espada que sostiene con la mano derecha y el manto que recoge con la izquierda completan los símbolos de la autoridad regia. Sobre la base de la escultura, se representa un casco de motivos fantásticos sobre el que el Emperador apoya su pierna, detrás el águila imperial. Se trata de una imagen muy relacionada con representaciones clásicas, denominadas en el Renacimiento "a la antigua", por las que se quería vincular el poder de Carlos V con el pasado imperial romano ${ }^{53}$. La escultura pública de Granada descansa sobre un gran pedestal cuadrangular, decorado con motivos geométricos, cuya base está formada por varios juegos de molduras. Los adornos que decoran las cuatro caras fueron copiados o realizados a juego con los situados en los pedestales de las columnas vecinas de la portada de la iglesia de San Justo y Pastor $^{54}$. Tenemos que recordar que cuando se ubica en esta plaza urbana la escultura el edificio que ocupa actualmente la Facultad de Derecho era, en realidad, la sede central de la Universidad de Granada. Eso ha significado que la plaza haya funcionado como lugar de encuentro de universitarios, de concentraciones y protestas, de celebraciones y reuniones. Estas funciones no siempre han tenido un sentido respetuoso y cordial, sino que con frecuencia esta escultura ha sido objeto de vandalismo, bien con carácter destructor o festivo, lo que no quita ninguna responsabilidad a los autores de los deterioros.

\section{CONCLUSIONES}

A modo de conclusiones señalaremos algunos aspectos que derivan de la visión conjunta de las esculturas expuestas. En cuanto a cronología todas se ubican a partir de 1948, correspondiendo aproximadamente el 50\% a realizadas en el siglo XXI. A nivel formal se mantienen formulas referidas a representaciones realistas, digamos, incluso, de carácter academicista, percibiéndose la estética contemporánea, sobre todo con rasgos

51 https://upload.wikimedia.org/wikipedia/commons/7/7b/Plaza_de_la_Universidad.JPG [consulta: 20 de diciembre de 2020].

52 Una segunda copia realizada en latón fundido y patinado para ubicarla en la Alhambra, finalmente pasó a la Universidad situándose en el centro del Patio del Archivo del Hospital Real. Cfr. https://patrimonio.ugr.es/ restauracion/restauracion-de-la-escultura-de-carlos-i-patio-del-archivo-hospital-real/ [consulta: 20 de diciembre de 2020].

53 Cfr. https://www.museodelprado.es/coleccion/obra-de-arte/carlos-v/c5d4a460-f4e2-4a39-8af0-240e180ab525 [consulta: 6 de diciembre de 2020].

54 La escultura fue pagada por el Ministerio de Educación Nacional y el basamento por el Ayuntamiento de la ciudad. 
expresionistas, en esculturas de los últimos 20 años. La abstracción como opción estética solo está presente cuando se trata de una obra ajena a la universidad en su concepción que después se integró en sus espacios ("Estudio para escultura urbana"). Los títulos de estas derivan de la identificación del personaje representado, solo existen tres con referentes conceptuales (“La danza”, “Desnudo femenino” y “Al investigador”).

En cuanto a los lugares de ubicación son interesantes señalar los puntos de contacto entre los espacios universitarios y los correspondientes a la ciudad, en un intento de proyectarse la institución educativa hacia la ciudadanía. También los espacios de acceso a edificios en distintos campus son lugares utilizados, estando a medio camino entre espacio público y privado (entendiendo este término referido a la comunidad universitaria). Los ejemplos menos frecuentes son los que están en el interior de edificios y cuando lo hacen están en lugares de acceso fácil y público, por lo que pueden ser observados por más personas que las estrictamente universitarias.

Hemos planteado el término itinerarios para su conocimiento, en tanto que pensamos en el valor didáctico y formativo de este recorrido tanto para los universitarios como para la ciudadanía en general, pudiéndose imbricar su lectura con la memoria de la universidad y la ciudad; así como para el estudiantado de materias relacionadas con la historia del arte y las Bellas Artes como vía de conocimiento directo de poéticas y técnicas.

La labor educativa que suponen estos itinerarios seguramente permitirán disminuir el vandalismo que han sufrido algunas de estas esculturas históricamente, siendo la más perjudicada la de Carlos V de la plaza de la Universidad ${ }^{55}$ que se ha tenido que someter a varios procesos de restauración, incluyendo reposición de partes perdidas y limpieza de pintura, lo que no quita que las autoridades responsables de este museo al aire libre cuiden de su conservación atendiendo al natural deterioro de carácter climático y el inexorable paso del tiempo.

Las esculturas tratadas en este trabajo no abarcan la totalidad de las existentes, aunque sí las más significativas y visibles. Por tanto, siendo una aproximación valiosa, la relación propuesta no permite la construcción de una memoria integral de la Universidad en un sentido amplio, sino mas bien la definición de hitos que marcan momentos aislados que son la historia de la Universidad, pero seleccionando capítulos dispersos.

55 Anualmente se realizaban actos vandálicos, a modo de novatadas universitarias, por parte de los estudiantes de la Facultad de Medicina en relación con los actos conmemorativos de San Lucas el 18 de octubre. 